\title{
DISCURSO DEL RECTOR DE LA UNIVERSIDAD DE CHILE
}

\author{
LUIS A. RIVEROS
}

Como miembro del Consejo Universitario, como Delegado Universitario y finalmente como Rector de la Universidad de Chile, Domeyko se empeñó en realizar importantes reformas en la estructura de la Corporación creada por Bello para que ésta incorporara la función docente. Con esto no pretendía suprimir ni menoscabar la tarea científica, que había sido tan central al concepto de Universidad concebido por Andrés Bello, sino por el contrario, se animaba a conseguir una articulación efectiva entre investigación y docencia, logrando que ambas se potenciaran. Esto fue decisivo para cumplir los propósitos de la Universidad en cuanto a formar a las elites dirigentes; crear no sólo conocimientos, sino además un pensamiento propio para "no estar siempre condenados a repetir servilmente las lecciones de la ciencia europea" -en el decir de Andrés Bello- y traspasar a la sociedad los beneficios de la ciencia por medio de una activa educación.

En términos generales, Domeyko sostenía como modelo a la universidad alemana, donde el profesor no se constituía en un repetidor de conocimientos obtenidos en revisiones bibliográficas, sino que formaba a los alumnos sobre la base de los resultados de sus propias investigaciones. Esto permitía transmitir los procesos de creación del conocimiento, otorgaba a la investigación un impulso de gran importancia. En su Memoria, presentada al Consejo de la Universidad el 4 de octubre de 1872, Domeyko anotaba: "la ventaja esencial que se saca de los estudios bien ordenados, ya sean preparatorios, ya superiores, es que se aprende a pensar y racionar: de manera que en cualquier situación de la vida en que se halle después el hombre sabrá estudiar, sabrá recordar y proseguir el cultivo de los ramos que le sean más necesarios y útiles"'.

'Domeyko, Ignacio, Reseña de los Trabajos de la Universidad desde 1855 hasta el presente. Memoria presentada al Consejo de la Universidad en sesión de 4 de octu- 
La creación de la Universidad de Chile en 1842, como universidad estatal y científica, en reemplazo de la colonial Universidad de San Felipe, real, docente y de claustro, es parte de un vasto proceso de secularización y modernización, en el que se valora las nuevas ciencias naturales y exactas por su utilidad práctica, en lugar del valor puramente especulativo e intelectual de la escolástica. Este proceso de construcción de la Universidad moderna en el país, fue liderado por dos grandes figuras: Andrés Bello e Ignacio Domeyko.

A Domeyko no lo convencía la eficacia de una universidad como la chilena, alejada de la función docente. Como ya se ha dicho, estaba convencido que el profesor era el profesional más indicado para producir conocimientos. Por lo tanto, debían ser los profesores de cada ramo los miembros de las facultades.

Fl sabio veía así el panorama de la Educación Superior en Chile: "Los miembros de las cinco facultades tenían el monopolio exclusivo de los exámenes, de los textos y del otorgamiento de los grados universitarios; debían dedicarse al progreso de las ciencias y de la literatura, pero ellos mismos no dictaban clases ni hacían cursos algunos en la universidad. Todas las ramas de las ciencias especiales como leyes, medicina, matemáticas superiores y ciencias naturales tenían sus cátedras (por lo demás muy escasas) en el llamado Instituto Nacional. En este plantel todos los cursos de instrucción superior estaban confundidos con los cursos de liceos y los elementales, todos los profesores y alumnos estaban bajo la autoridad de un solo rector y sujetos a iguales normas de disciplina y orden, casi sin considerar la edad ni el carácter de los estudiantes..."2.

En 1846, terminado el plazo de su contrato, Domeyko se preparaba para volver a Europa, pero el Ministro de Instrucción Manuel Montt le pidió que se quedara en Santiago por un par de meses para presentarle al Consejo Universitario un plan de reformas. "El proyecto presentado por mí al Consejo de Instrucción Pública -anota el sabio- consistía principalmente en que se separase del Instituto las cátedras correspondientes de la instrucción superior y, una vez completadas con otras

bre de 1872 por el señor Rector, don Ignacio Domeyko. Santiago, Imprenta Nacional, 1872.

2 I)omeyko, Ignacio, Mis viajes. 
ranlas científicas indispensables que aún faltaban, se creara con ellas una universidad similar a las alemanas..."3.

A principios de 1847 el Consejo Universitario aprobó la reforma propuesta por Domeyko. El 22 de noviembre de ese año se dictaba un nuevo plan de organización de los estudios universitarios, el que sólo vendría a ponerse en práctica en 1852. Por él se dispuso que el Instituto se dividiría en dos secciones: una destinada a la instrucción secundaria o preparatoria, que conservaría el nombre de Instituto, quedando bajo la dirección de un rector. La otra, dedicada a la enseñanza universitaria o profesional y científica, sería dirigida por un Delegado universitario. Domeyko recuerda que "Se acordó también, por cortesía a Antonio Varas (quien se había opuesto a la reforma) no darle todavía a esta segunda sección el nombre de Universidad, sino de Delegación" ".

El trámite no había sido fácil. Como se puede apreciar, pasaron casi 5 años, entre 1847 y 1852 para que se cumpliera el acuerdo del Consejo. Lo que precipitó este hecho fueron los disturbios estudiantiles que se produjeron en el Instituto como protesta por lo que se decía constituía un extremo rigor escolar. Entonces se le ofreció el cargo de Delegado Universitario a Domeyko, quien puso como condición que se le permitiera "separar de la escuela principal, es decir del Instituto, todas las clases de instrucción superior (o sea leyes, medicina y matemáticas, confundidas hasta ahora con las secundarias) y organizar con estas clases tres facultades similares a las universidades alemanas, bajo mi dirección provisoria inmediata". Pidió además que los estudiantes de estas facultades quedaran libres del rigor escolar, que había sido la causa de los disturbios.

El gobierno accedió a cumplir con estas condiciones, y así, a principios de marzo de 1852 se designó en el cargo de Delegado universitario a Ignacio Domeyko, quien lo ejerció hasta el momento en que es elegido rector, el 6 de septiembre de 1867.

En su memoria del año 1861, Domeyko informaba sobre los avances de los estudios superiores desde la reestructuración: se había duplicado el número de clases, de profesores y de alumnos, organizándose los estudios para las profesiones científicas radicadas en tres faculta-

\footnotetext{
${ }^{3}$ Ihid.

I Ibiel.
} 
des en forma de escuelas superiores especiales: la jurídica, la de medicina y la politécnica. Esta última formaba a los ingenieros civiles y de minas. Bajo su supervisión directa quedaba, además, la facultad de Bellas Artes, que descle 1858 formaba parte de la sección universitaria del Instituto. La atención que Domeyko prestó a sus escuelas, que eran de pintura, escultura y arquitectura, fue igual que la que ponía en el perfeccionamiento de los estudios científicos5.

Una vez nombrado Delegado, Domeyko inició de inmediato la reorganización de la instrucción superior, elaborando un nuevo programa para medicina y ciencias físicas y matemáticas que sometió a la consideración de las comisiones de cada facultad, mientras a leyes le pidió que propusiera su propia reforma. Ellas fueron de gran importancia pues establecieron un orden obligatorio de estudios, crearon nuevas cátedras y se fundaron las ingenierías.

En 1867, como se ha dicho, Ignacio I omeyko es elegido rector. "Recáían en mí el título y la autoridad que ejerció por más de veinticinco anoos Andrés Bello, el patriota más enıdito y más estimado en toda América española" -recuerda el sabio polaco-. Entonces se suprime la Delegación universitaria, puesto que a partir de entonces el rector se ocupa directamente de la instrucción superior y de la administración de la docencia universitaria.

Fue tan fecundo este primer quinquenio rectoral, que sin ni siquiera preguntarle su opinión, lo eligieron casi por unanimidad para un segundo período. Incluso el adversario más enconado a sus proyectos de reforma, Antonio Varas, concurrió a votar por él. En este segundo período debió enfrentar el primer ataque al Estado docente que ocurre cuando el Ministro de Instrucción Abdón Cifuentes decide implantar la ilimitada libertad de enseñanza, casi sin control estatal y el libre acceso a las profesiones de médico, abogado, profesor, ingeniero, etc.

Las observaciones sobre los efectos de esta situación que hace Ignacio IDomeyko son muy certeras y conviene reproducirlas: "La porfía del Ministro me produjo no pocos contratiempos. Porque no tardaron en formarse numerosas escuelitas mantenidas por especuladores, en donde, por cualquier retribución, se entregaban, se vendían certificados, con los que llegaban a la Universidad ignorantes, pero también aquí los exá-

"Mellafe, Rolando et al., Historia de la Lniversidad de Chile. Ediciones de la Universidad de Chile, Biblioteca Central. Santiago, 1992. 
menes se trocaban en pura fórmula. También para las ramas superiores de la ciencia se fundaban escuelas privadas, algunas con el propósito de propagar principios inmorales, otras con afán de lucro y miras políticas. Bajo la apariencia de escuela apareció un verdadero bazar de venta de certificados, que emitió, en un solo año, hasta quinientos certificados de cursos efectuados y de exámenes rendidos donde ni siquiera hubo profesores (...) Las nuevas disposiciones liberales del Ministro dieron origen al desorden en los liceos fiscales, se tradujeron pronto en una decadencia de los cursos universitarios y en ningún caso contribuyeron al desarrollo de mejores escuelas particulares, laicas y religiosas"6. Sin ninguna duda, el fondo de estas observaciones se podría aplicar con mucha justicia a la situación actual de muchos países en que ha empezado a prevalecer un total libre mercado en la enseñanza.

Aún cuando hizo presente su avanzada edad, su intención de regresar a su tierra natal y algunos problemas de salud, Domeyko fue elegido rector por un tercer período. En éste se consagra estatutariamente, con la ley orgánica del 9 de enero de 1879, la función docente de la Universidad. Durante ese período Chile entró en la Guerra del Pacífico. Domeyko recuerda: "La enseñanza se resintió al ingresar gran parte de los estudiantes universitarios en el ejército. Yo aprobaba su prontitud para ponerse al servicio de la patria, su voluntad y su valor, y probablemente animé a más de uno de los mayores a tomar las armas, pero la guerra se prolongó mucho y la atención de la juventud dirigida hacia ella causaba cierta desorganización en las clases universitarias"7.

A mediados de 1882 , se acercaban las nuevas elecciones de rector, y Domeyko presenta con anticipación su solicitud de relevo de sus obligaciones docentes, de rector y la de su jubilación. Sin embargo se produjo un problema político insoluble y Domeyko terminó siendo elegido nuevamente rector. Tenía 80 años y problemas crecientes de audición. Asumió el cargo sólo con el compromiso que se aceptaría su dimisión dentro de un tiempo prudente, y entonces el gobierno ordenaría nuevas elecciones.

De modo que el sabio estuvo un año más en el cargo de rector. Recuerda que a comienzos de marzo de 1883 inició por última vez sus

\footnotetext{
${ }^{6}$ Domeyko, Ignacio, Mis viajes.

7 Ibid.
} 
clases de química y mineralogía. Resulta conmovedora su devoción por la docencia: "Tuve ese año más de cien alumnos en química y veinte en mineralogía, siendo las clases diarias al mediodía, éstas no me cansaban, tenían para mí siempre el mismo encanto. Sólo me aburrían y molestaban las sesiones semanales del Consejo Universitario, en las que no lograba oír a los padres y decanos sentados cerca de mí, causándome, además, dolor de ojos las luces parpadeantes de gas carbónico de la inmensa araña colgada delante de mí"s.

Llegó finalmente el retiro. El sabio cumplió su viejo y postergado deseo de volver a Polonia, luego viajó por Europa desde donde regresó a Chile poco antes de morir.

Ignacio Domeyko es uno de los maestros fundadores de la Universidad de Chile y uno de sus grandes rectores. Las reformas fundamentales que realizó permitieron el desarrollo posterior de la Corporación y sus grandes logros, como la creación del Instituto Pedagógico, en 1889, para dar finalidad docente a la Facultad de Filosofía y Humanidades; el Estatuto de 1931; el desarrollo armónico de la docencia y la investigación; la profesionalización de la labor del científico.

La figura de Ignacio Domeyko constituye un paradigma del ser universitario, y lo más representativo de la vocación docente del verdadero académico. Fue uno de los más insignes promotores del cumplimiento de la misión nacional de la Universidad de Chile que con orgullo cultivamos hasta hoy: "Generación y diseminación de conocimiento para el desarrollo integral del país". En mi condición de Rector de la Universidad de Chile, es para mi grato y emocionante rendirle este homenaje sincero y sentido a nombre de la institución que represento.

$Y$ es que la presencia de Ignacio Domeyko trasciende al tiempo y al espacio. En realidad, su figura se pasea por el amplio jardín interior de la casa, por sus vetustos aleros, por las habitaciones cargadas de tiempo, y por el ánimo de quienes la habitan, tan vinculado a la memoria del pasado y del glorioso antepasado. Al recordar en esta sala mi vivencia en la vieja casa de calle Cueto -vinculada por azar a mis más tempranos recuerdos de niño- solo quiero reseñar lo trascendente que puede resultar una persona, lo inmutable en el tiempo que puede ser su figura, y lo significativo que esa misma persona haya sido un gran académico y un gran rector de una gran universidad. Ha sido muy

"Ibid. 
rico para mi descubrir al hombre y acercarme a sus vivencias más cotidianas, y conocer de cerca los retos que su existencia ha dejado para quienes hemos continuado en la senda académica.

Estuve en su hogar en Santiago. Visité su escritorio y toqué sus anaqueles de libros, entre los cuales figura una colección de la revista Anales de la Universidad de Chile, como también libros especializados en su pasión de vida: el estudio de los minerales. Se respiraba en el lugar un aire de recogimiento; como si la presencia del sabio se sintiera aún en la vieja casa de la calle Cueto; en la esquina del cuarto, su foto tomada en la tierra natal, en una visita que efectuara cuando ya era un hombre maduro. Por doquier, sus adornos, el escudo polaco y aún restos de su colección de minerales -pese a que el grueso de la misma se encuentra en la Universidad de La Serena.

Su familia me ha hablado del Ignacio Domeyko hombre, padre, abuelo, bisabuelo. Del Ignacio Domeyko inmigrante, profesor, investigador, rector y sabio insigne. De su familia se puede desprender la presencia siempre viva de la memoria del ilustre fundador de la dinastía. Desde aquí, su segundo hogar de siempre, podemos hablar del Domeyko cientista, docente, humanista que adorna con excelencia el ilustre pasado de esta Universidad. 